\title{
Structural Dynamics of Large Space Structures Having Random Parametric Uncertainties
}

\author{
Robert M. Koch \\ Naval Undersea Warfare Center Division, 1176 Howell Street, Code 8232, Bldg. 1302, Newport, RI 02841-1708 USA
}

(Received 19 February 2001; accepted 15 January 2003)

\begin{abstract}
Deployable periodic large space structures have been shown to exhibit high dynamic sensitivity to periodbreaking imperfections and uncertainties. These can be brought on by manufacturing or assembly errors, structural imperfections, as well as nonlinear and/or nonconservative joint behaviour. In addition, the necessity of precise pointing and position capability can require the consideration of these usually negligible and unknown parametric uncertainties and their effect on the overall dynamic response of large space structures. This work describes the use of a new design approach for the global dynamic solution of beam-like periodic space structures possessing parametric uncertainties. Specifically, the effect of random flexible joints on the free vibrations of simply supported periodic large space trusses is considered. The formulation is a hybrid approach in terms of an extended Timoshenko beam continuum model, Monte Carlo simulation scheme, and first-order perturbation methods. The mean and mean-square response statistics for a variety of free random vibration problems are derived for various input, random joint stiffness probability distributions. The results of this effort show that, although joint flexibility has a substantial effect on the modal dynamic response of periodic large space trusses, the effect of any reasonable uncertainty or randomness associated with these joint flexibilities is insignificant.
\end{abstract}

\section{NOMENCLATURE}

$A_{c} \quad$ - chord member cross-sectional area

$A_{d} \quad$ - diagonal member cross-sectional area

$A_{g} \quad$ - girder member cross-sectional area

E - Young's modulus

$E\{\quad$ - expectation operator

$E A \quad$ - beam axial rigidity

EI - beam flexural rigidity

$G A$ - beam shear deformation rigidity

$k \quad-$ joint spring stiffness parameter

$L \quad$ - beam overall length

$L_{c} \quad$ - chord member length

$L_{g} \quad$ - girder member length

$M \quad-$ moment stress resultant or total number of cells in truss structure

$N \quad$ - axial stress resultant

$Q \quad-$ transverse shear stress resultant

$r \quad$ - correlation coefficient

$T \quad-$ time

$u(x, t) \quad$ - beam axial deflection

$w(x, t)$ - beam transverse deflection

$x \quad$ - beam spatial variable, $x=0$ at left end of beam

$z \quad-$ beam spatial variable in transverse direction

$\varepsilon \quad-$ perturbation coefficient

$\eta_{i j} \quad-$ coupling stiffness coefficients

$\eta_{i j}^{*} \quad-$ coupling flexibility coefficients

$\lambda_{n} \quad-$ ratio of $\Psi / W$ amplitudes for the $n$-th mode

$\mu \quad-$ mean value of random variable (r.v.)

$\rho \quad-$ mass density

$\rho A \quad-$ beam mass per unit length

$\rho I \quad$ - beam mass moment of inertia per unit length

$\sigma \quad-$ standard deviation of random variable

$\sigma^{2} \quad-$ variance of random variable

$\sigma / \mu \quad-$ ratio of standard deviation to mean of a r.v.

$\psi(x, t)$ - beam slope due to bending only (eliminating shear effects)

$\omega_{n} \quad$ - beam's $n$-th axial or flexural natural frequency

\section{INTRODUCTION}

The recent interest in the dynamic structural analysis of large space trusses has evolved principally due to their proposed usage in future high-orbit, near-zero-gravity space applications. In addition, the development of reusable space transportation such as the space shuttle offers the ability to transport sections of these large space structures to outer space and assemble them there. Thus, deployable space trusses, with their relatively low cost, low weight, high stiffness, and large size capability, prove to be ideal for this application; in particular, skeletal periodic or repetitive beam-like truss structures have been identified as potential candidates for the proposed NASA space station.

These prefabricated truss structures, after being condensed and shipped into orbit, must be quickly and efficiently assembled in order to minimise dangerous astronaut exposure time. Hence, quick-locking joints, designed to rotate and lock in place, have been developed. Unfortunately, however, these joints introduce additional complexities to the dynamic and control analysis of large space trusses that do not exist in trusses consisting of so-called ideal rigidly pinned joints; most notable of these are nonlinearities such as hysteretic or passive damping, gap effects, and unknown joint flexibilities. Furthermore, their properties vary from joint to joint in some complicated manner, offering little hope for an accurate closed-form deterministic solution.

Due to the large number of structural elements present in such beam-like truss structures, it is convenient to evaluate their global or gross dynamic response by representing them as equivalent elastic continua. ${ }^{1}$ That this approach is indeed viable has been illustrated in a series of papers in which the frequencies of periodic beam-like truss structures were obtained from an equivalent beam model. ${ }^{2-4}$ In an effort to extract local member characteristics from the global continuum beam model's dynamic behaviour, a global-local approach was recently proposed. ${ }^{5}$ In all of these studies, the equivalent beam stiffness properties are extracted from a typical cell by

International Journal of Acoustics and Vibration, Vol. 8, No. 2, 2003 (pp 95-103) 